\title{
Optimum combined test plans for systems and components
}

\author{
JAYANT RAJGOPAL, MAINAK MAZUMDAR and SUBBA RAO V. MAJETY \\ Department of Industrial Engineering, 1048 Benedum Hall, University of Pittsburgh, Pittsburgh, PA 15261, USA \\ E-mail: rajgopal@engrng.pitt.edu; mmazumd@engrng.pitt.edu; majety+@engrng.pitt.edu
}

Received February 1997 and accepted April 1998

\begin{abstract}
Since mathematical models based on component reliabilities are frequently used for prediction of system reliability, it stands to reason that cost-effective inferences on the reliability of a system could be made on the basis of tests of its constituent components. Prior research in the area of system-based component testing has for the most part addressed the development of plans that test only the components. From a practitioner's point of view, this is an issue of concern since system failures are often caused by imperfect interfaces and other causes that are not directly attributable to component failures. The exclusion of system tests may thus be an erroneous approach. This paper addresses the development of test plans that explicitly consider the possibility of interface failures. The paper analyzes a series system to determine when testing should be performed on the system alone, on the components only, and on both, depending on test costs and interface reliabilities. Optimum test plans are derived by solving a twostage mathematical program.
\end{abstract}

\section{Introduction}

An important step in the development of any system is a program of testing to ensure that the system will meet some specified reliability level. Such tests can often be quite expensive and thus one commonly looks for minimum cost test plans. The constraints to be satisfied by such plans are usually in the form of maximum limits on the probability of both Type 1 and Type 2 errors. A typical test plan may be found for instance in the Department of the Navy document MIL-HDBK-781 [1]. Here a user must specify values for $\alpha$ (the maximum acceptable producer's risk), $\beta$ (the maximum acceptable consumer's risk), $R_{0}$ (a value such that a system with a reliability less than $R_{0}$ over its mission time would be considered definitely unacceptable), and $R_{1}$ (a value such that a system with a reliability higher than $R_{1}$ over its mission time would be considered definitely acceptable), where $0<R_{0}<R_{1}<1$. The two constraints that should be met by a test plan may be stated as follows:

- Pr (Plan rejects the system | System reliability over its mission time $\left.\geq R_{1}\right) \leq \alpha$.

- Pr (Plan accepts the system $\mid$ System reliability over its mission time $\left.\leq R_{0}\right) \leq \beta$.

In practice, few people actually approach this problem in a systematic fashion, with the explicit objective of minimizing total test costs, and the methods used tend to be of an ad hoc nature. In fact, there may be many different test plans that meet the above constraints, each with its own procedure and its own acceptance criterion, and one would like to choose from these the plan that is the least expensive in terms of total test costs. However, since it is clearly impossible to arrive at an exhaustive list of all feasible test plans, a general approach may be to decide upon some class of plans that is plausible for the specific system under consideration.

In developing test plans for systems, there are two basic approaches: (1) system tests where the entire system is assembled and tested; and (2) component tests where only the components are tested. In practice, one would perform a combination of both. System tests are appropriate where component failures are not independent of each other, interfaces between components are unreliable or the systems themselves are relatively inexpensive and easy to test. On the other hand, component test plans can offer significant advantages when conditions differ from the above. They are almost always cheaper than system level tests and less complicated in terms of instrumentation and equipment. Moreover, component tests can be conducted at different locations and times and since the entire system need not be assembled prior to testing, the development of the system tends to be much more timely and inexpensive. These and other advantages of component test plans are discussed by Rajgopal and Mazumdar [2].

Component testing is quite common in practice and the plans that are used are usually well-designed from a component reliability demonstration perspective. However, they are usually not system-based, i.e., they are not explicitly designed with system reliability estimation or demonstration in mind. As a result, the actual protection 
offered by these plans in making inferences about system reliability is not the same as what is advertised; this point is further discussed by Easterling et al. [3]. A significant proportion of research in the area of component testing has concentrated on the design of statistically valid, system-based component test plans that also have minimum cost. Test plans for various system configurations have been studied by several authors. Gal [4] appears to have been the first to write on this topic. He was followed by others including Mazumdar [5,6], Yan and Mazumdar [7,8], Rajgopal and Mazumdar [9-10,11], Easterling et al. [3], and Altinel $[12,13]$. All of these papers demonstrate how component tests should be designed from a system perspective. While these papers address various issues related to the design of system-based component test plans, none of them explicitly account for failures of the interfaces between components. From the point of view of a practitioner, this is a serious drawback since it is common knowledge that many systems often fail due to reasons other than component failures; an illustration of this is provided by Denson [14] in his examination of 16 different defense systems for the root cause of system failure, and by Murphy and Gent [15] who look at software reliability. Many practitioners are thus uncomfortable with completely forgoing system testing and relying solely on component tests for the verification of system reliability. One paper that does address combined system and component testing is that written by Easterling and Prairie [16]; however, this paper considered the case of a system with $m$ identical components and was written with a view to estimating component reliabilities as opposed to explicit demonstration of system reliability.

In this work we address for the first time the issue of interfaces in the context of component test plans, and also the issue of combining system testing with component level tests. The term "interface" is used here in a broad sense and failure due to an imperfect interface is defined as any system failure that cannot be directly attributed to the failure of a constituent component. We restrict ourselves to a series system with exponentially distributed failure times so that the exposition is simple, but future work will obviously have to address other system configurations and other failure time distributions. For the series system considered in this paper, the design of the test plans may be formulated as a mathematical program for which the solution is obtained by using statistical properties of the Poisson distribution and solving linear programs in two stages.

It should also be noted that occasionally it might be possible to test the interface itself, e.g., when the interface is a mechanical linkage or a connecting wire. In such instances, one could treat the interface as just one more component of the system and thus the previous work in this area would be applicable. However, in many other cases, such an approach may be infeasible, e.g., when the interface is a weld or a solder, or even for one of the above examples. In such situations the only way to evaluate the effect of the interface on system reliability is to test the entire system, and it is such situations that we consider in this paper.

\section{Combined system and component tests}

Consider a system of $n$ independent components in series. It is assumed that:

- The failure time of component $j$ is an exponentially distributed random variable with (an unknown) mean equal to $\lambda_{j}^{-1}$.

- The sum of the component failure rates is defined by $\lambda_{C}=\sum_{j} \lambda_{j}$.

- The interfaces between the components are imperfect and together have a failure time that is an exponentially distributed random variable with (an unknown) mean equal to $\lambda_{I}^{-1}$.

- Component and interface failures are mutually independent.

- Without loss of generality, the mission time is equal to one unit of time so that the reliability of the system, $R_{S}=\exp \left(-\lambda_{I}-\lambda_{C}\right)$.

- Two numbers $R_{0}$ and $R_{1}\left(0<R_{0}<R_{1}<1\right)$ are specified such that the system is considered definitely unacceptable if $R_{S} \leq R_{0}$ and definitely acceptable if $R_{S} \geq R_{1}$.

- Two small fractions $\alpha$ and $\beta$ corresponding to the maximum acceptable levels of Type I and Type II error respectively are specified, with $\alpha+\beta<1$.

- It costs $c_{j}$ units to test component $j$ for 1 unit of time, while the cost of a system level test is $c_{S}$ units per unit time, where the unit of time is the mission time.

The following test plan is in effect: each component $j$ is tested (with replacement of failed components) for $t_{j}$ units of time, and the system as a whole is tested (again with replacement of failed systems) for $t_{S}$ units of time. Suppose $X_{j}$ represents the number of failures observed for component $j$ and $X_{S}$ the number of system failures. It is assumed that $X_{1}, X_{2}, \ldots, X_{n}$ and $X_{S}$ are mutually independent random variables. The statistical problem that we address is equivalent to a test of the null hypothesis $H_{0}: R_{S} \leq R_{0}$ against the alternative $H_{1}: R_{S} \geq R_{1}$ such that the probability requirements on the two types of error are satisfied, and the total test costs are minimized. It is not immediately obvious how the globally optimum critical region can be characterized in this situation, and one could consider adopting different approaches for obtaining a suitable critical region based on the sample observations. One possibility is the use of tests based on the maximum likelihood estimates or the likelihood ratio criterion. Here we confine ourselves to an $a d-h o c$ class of critical regions constructed according to the "sum rule" which operates as follows: if the total number of failures 
$X\left(=X_{1}+X_{2}+\cdots+X_{n}+X_{S}\right)$ does not exceed some integer $m$ the system is accepted, otherwise the system is rejected. This rule has been used in the past for series systems (e.g., Altinel [13], Easterling et al. [3], and Rajgopal and Mazumdar [10]). It is assumed that system failures are not specifically classified as to whether they are caused by component failures or by interface failures. While this information could be used to make the test plans more efficient, the requisite mathematical development is beyond the scope of this paper.

We consider the suggested testing scheme along with the sum rule in the current context because this leads to tractable mathematical derivations and allows us to obtain an understanding of the issues related to allocation between system and component testing. However, other ad-hoc testing schemes and acceptance rules are certainly plausible. For instance, one could use the following rule: accept the system if $X_{1}+X_{2}+\cdots+X_{n} \leq m_{C}$ and $X_{S} \leq m_{S}$, where $m_{C}$ and $m_{S}$ are constants to be determined. Similarly, one could use a weighted sum rule with the magnitude of the weights depending upon the observed relative magnitudes of the component and interface failure rates. However this would require some form of approximation since the resulting distributions to be analyzed are no longer Poisson. The use of other, more efficient testing schemes and acceptance rules are certainly open issues.

A primary result that will considerably simplify the exposition that follows is that in the absence of any prior information on the relative magnitudes of the component failure rates, if components are to be tested then all components must be tested for the same amount of time $t_{1}=t_{2}=\cdots=t_{n}=t_{C}$. This is irrespective of component test costs. The result is a consequence of the fact that a series system is only as good as its weakest link and thus with no a priori information on component reliabilities or failure rates, there is no justification for testing one component for less time than another. Put another way, since the precision of the estimate of system reliability is driven by the minimum time on test across all components, testing one component more than another only adds to the cost without any addition in precision. This fact has been proved in several papers relating to tests on series system (e.g., Yan and Mazumdar [7], Rajgopal and Mazumdar [10,11], Altinel [12] etc.). Rather than prove it again, we will simplify the problem by denoting the common test time for each component as $t_{C}$ in the development that follows.

In deriving a minimum cost test plan based on the sum rule, the questions of interest are:

- Under what conditions on the values of $c_{j}$ and $c_{S}$ is it optimal to test: (a) only the components; (b) only the system; and (c) both components as well as the system?
- What are the optimum values of $t_{C}$ and $t_{S}$ ?

- What is the optimum value of $m$ ?

We address these questions by considering in turn, two cases:

(1) The magnitude of $\lambda_{I}$ is completely unknown.

(2) Some a priori information on the value of $\lambda_{I}$ is available.

In both cases, the generic optimization problem may be stated as:

$$
\text { Minimize } C=c_{S} t_{S}+\left(\sum_{j=1}^{n} c_{j}\right) t_{C},
$$

subject to

$$
\begin{gathered}
\operatorname{Pr}\left\{X_{S}+\sum_{j=1}^{n} X_{j} \leq m \mid R_{S} \geq R_{1}\right\} \geq 1-\alpha, \\
\operatorname{Pr}\left\{X_{S}+\sum_{j=1}^{n} X_{j} \leq m \mid R_{S} \leq R_{0}\right\} \leq \beta, \\
t_{S}, t_{C} \geq 0 .
\end{gathered}
$$

We now introduce some notation. First, define $F_{m}(\lambda)$ as the distribution function of a Poisson random variable $Y$ with mean $\lambda$, i.e., $F_{m}(\lambda)=\operatorname{Pr}(Y \leq m)$. Also, given $0 \leq \gamma \leq 1$, define $\phi_{m}(\gamma)$ as the mean of a Poisson random variable $Y$ for which $\operatorname{Pr}(Y \leq m)=\gamma$, i.e.,

$$
\begin{aligned}
F_{m}\left(\phi_{m}(\gamma)\right)= & \gamma=\exp \left(-\phi_{m}(\gamma)\right) \\
& \times\left[1+\phi_{m}(\gamma)+\frac{\left(\phi_{m}(\gamma)\right)^{2}}{2 !}+\cdots+\frac{\left(\phi_{m}(\gamma)\right)^{m}}{m !}\right] .
\end{aligned}
$$

Given $m$ and $\gamma$, the value of $\phi_{m}(\gamma)$ is easily computed by solving the above nonlinear equation using a simple technique such as the Newton-Raphson method. The authors have computed and tabulated the values of $\phi_{m}(\gamma)$ for various values of $\gamma$ between 0 and 1 and for values of $m$ ranging from 0 through 600 ; this table of values is available from the authors on request. Note that the value of $\phi_{m}(\gamma)$ decreases with $\gamma$ (for fixed $m$ ) and increases with $m$ (for fixed $\gamma$ ).

\subsection{No prior information}

In this section we assume that there is no knowledge about the magnitude of $\lambda_{I}$ relative to the $\lambda_{j}$ values. Now, since $X_{S}$ is Poisson with a mean $t_{S}\left(\lambda_{I}+\lambda_{C}\right)$ and each $X_{j}$ is Poisson with a mean $t_{C} \lambda_{j}$, it is clear that $X_{S}+\sum_{j} X_{j}$ is Poisson with a parameter $t_{S}\left(\lambda_{I}+\lambda_{C}\right)+t_{C} \lambda_{C}=t_{S} \lambda_{I}+$ $\left(t_{S}+t_{C}\right) \lambda_{C}$. The system reliability is given by $R_{S}=\exp \left(-\lambda_{I}-\lambda_{C}\right)$. Using (4), the constraints (2) and (3) may then be rewritten respectively as 


$$
\begin{aligned}
& F_{m}(\Lambda) \geq F_{m}\left(\phi_{m}(1-\alpha)\right) \text { for } \\
& \quad\left\{\lambda_{I}, \lambda_{C} \mid \exp \left(-\lambda_{I}-\lambda_{C}\right) \geq R_{1} ; \lambda_{I}, \lambda_{C} \geq 0\right\} \\
& F_{m}(\Lambda) \leq F_{m}\left(\phi_{m}(\beta)\right) \text { for } \\
& \quad\left\{\lambda_{1}, \lambda_{C} \mid \exp \left(-\lambda_{I}-\lambda_{C}\right) \leq R_{0} ; \lambda_{I}, \lambda_{C} \geq 0\right\}
\end{aligned}
$$

where

$$
\Lambda=t_{S} \lambda_{I}+\left(t_{S}+t_{C}\right) \lambda_{C} .
$$

Since the Poisson distribution function $F_{m}(\gamma)$ is strictly decreasing in $\gamma$, Equation (7) may be used to rewrite (5) and (6) as

$$
\begin{aligned}
& t_{S} \lambda_{I}+\left(t_{S}+t_{C}\right) \lambda_{C} \leq \phi_{m}(1-\alpha) \text { for } \\
& \quad\left\{\lambda_{I}, \lambda_{C} \mid \lambda_{I}+\lambda_{C} \leq-\ln R_{1} ; \lambda_{I}, \lambda_{C} \geq 0\right\} . \\
& t_{S} \lambda_{I}+\left(t_{S}+t_{C}\right) \lambda_{C} \geq \phi_{m}(\beta) \text { for } \\
& \quad\left\{\lambda_{I}, \lambda_{C} \mid \lambda_{I}+\lambda_{C} \geq-\ln R_{0} ; \lambda_{I}, \lambda_{C} \geq 0\right\} .
\end{aligned}
$$

Note that (8) and (9) are defined for fixed nonnegative values of $t_{C}$ and $t_{S}$ and are constraints derived from (2) and (3) respectively. Thus the LHS of constraints (2) and (3) reduce to solving the following two "inner" linear programming subproblems in $\lambda_{I}$ and $\lambda_{C}$ :

Subproblem 1: Maximize $t_{S} \lambda_{I}+\left(t_{S}+t_{C}\right) \lambda_{C}$, subject to

$$
\left\{\lambda_{I}+\lambda_{C} \leq-\ln R_{1} ; \lambda_{I}, \lambda_{C} \geq 0\right\} .
$$

Subproblem 2: Minimize $t_{S} \lambda_{I}+\left(t_{S}+t_{C}\right) \lambda_{C}$, subject to

$$
\left\{\lambda_{I}+\lambda_{C} \geq-\ln R_{0} ; \lambda_{I}, \lambda_{C} \geq 0\right\} .
$$

Let us now define

$$
\delta=\lambda_{I} /\left(\sum_{j=1}^{n} \lambda_{j}\right)=\lambda_{I} / \lambda_{C} .
$$

Note that $\delta$ provides a measure of the relative magnitudes of the failure rates of the interfaces and the components, and that since $\lambda_{I}$ and the $\lambda_{j}$ are unknown, so is $\delta$. Using $\lambda_{I}=\delta \lambda_{C}$ from (12) to eliminate $\lambda_{I}$ in (10) and (11), the overall "outer" optimization problem then is to find nonnegative values of $t_{C}$ and $t_{S}$ so as to

$$
\text { Minimize } C=c_{S} t_{S}+\left(\sum_{j=1}^{n} c_{j}\right) t_{C},
$$

subject to

$\left\{\right.$ Maximum $\left\{(1+\delta) t_{S}+t_{C}\right\} \lambda_{C}$, subject to

$$
\left.\left\{(1+\delta) \lambda_{C} \leq-\ln R_{1}, \lambda_{C} \geq 0\right\}\right\} \leq \phi_{m}(1-\alpha),
$$

$\left\{\right.$ Minimum $\left\{(1+\delta) t_{S}+t_{C}\right\} \lambda_{C}$, subject to

$$
\left.\left\{(1+\delta) \lambda_{C} \geq-\ln R_{0}, \lambda_{C} \geq 0\right\}\right\} \geq \phi_{m}(\beta),
$$

$$
t_{S}, t_{C} \geq 0 .
$$

It is clear that the optimum values of the two subproblems defining the LHS of constraints (14) and (15) are given by $\left(-\ln R_{1}\right)\left\{t_{S}+t_{C} /(1+\delta)\right\}$ and $\left(-\ln R_{0}\right)\left\{t_{S}+\right.$ $\left.t_{C} /(1+\delta)\right\}$ respectively. If we now define

$$
\begin{gathered}
A(m)=\phi_{m}(1-\alpha) /\left(-\ln R_{1}\right) . \\
B(m)=\phi_{m}(\beta) /\left(-\ln R_{0}\right) .
\end{gathered}
$$

then the optimization problem reduces to

$$
\text { Minimize } C=c_{S} t_{S}+\left(\sum_{j=1}^{n} c_{j}\right) t_{C},
$$

subject to

$$
\begin{gathered}
B(m) \leq\left\{t_{S}+t_{C} /(1+\delta)\right\} \leq A(m), \\
t_{S}, t_{C} \geq 0 .
\end{gathered}
$$

This is feasible for all $m$ such that $B(m) \leq A(m)$, i.e., $\phi_{m}(\beta) /\left(-\ln R_{0}\right) \leq \phi_{m}(1-\alpha) /\left(-\ln R_{1}\right)$, i.e., $\left(-\ln R_{1}\right) /$ $\left(-\ln R_{0}\right) \leq \phi_{m}(1-\alpha) / \phi_{m}(\beta)$. The LHS of this last inequality is strictly less than 1 , and it has been shown by Rajgopal et al. [17] that as long as $\alpha+\beta<1$, the ratio in the RHS is strictly increasing in $m$ and approaches 1 as $m$ approaches $\infty$. Thus, the problem is feasible for all $m \geq m^{*}$ where $m^{*}$ is defined as

$$
m^{*}=\operatorname{Inf}\left\{m \mid\left(-\ln R_{1}\right) /\left(-\ln R_{0}\right) \leq \phi_{m}(1-\alpha) / \phi_{m}(\beta)\right\} .
$$

Returning to our problem, for a feasible value of $m$ it is clear that at the optimum we must have $\left\{t_{S}+t_{C}\right\}$ $(1+\delta)\}=B(m)$, i.e., $t_{C}+t_{S}(1+\delta)=(1+\delta) B(m)$; the upper bounding constraint is redundant. Since the overall optimization problem is a linear program with a single constraint it follows that at the optimum either $t_{C}$ or $t_{S}$ must be equal to zero. A little examination shows that the optimum solution is given by

- $t_{S}=0, \quad t_{C}=(1+\delta) B(m), \quad$ with $C=\left(\sum_{j} c_{j}\right)(1+\delta)$ $B(m)$ if $c_{S} \geq(1+\delta)\left(\sum_{j} c_{j}\right)$.

- $t_{S}=B(m), \quad t_{C}=0, \quad$ with $\quad C=c_{S} B(m) \quad$ if $\quad c_{S} \leq$ $(1+\delta)\left(\sum_{j} c_{j}\right)$

Finally, since $\phi_{m}(\beta)$ is strictly increasing in $m$, so is $B(m)$. Thus the optimum value of $m$ is the smallest one that leads to a feasible problem, i.e., equal to $m^{*}$ as defined by (18).

In summary, for a series system with imperfect interfaces and no a priori knowledge on the relative magnitude of the interface failure rate, the optimal policy calls for testing either only the system or only the component. The specific policy will depend on $\delta$, i.e., on how the relative magnitudes of the system and component test costs compare with the relative magnitudes of the interface and component failure rates. Unfortunately, the value of $\delta$ is obviously unknown and the above results cannot be 
applied in any useful way. In the next section we consider test plans where some a priori knowledge about $\lambda_{I}$ is available. Before doing so we consider two special cases that offer some insight into the policy and its intuitive appeal.

First, consider the case when $\delta=0$, i.e., the interfaces are perfect. Then if the total cost of testing the components is less than that of testing the system, the optimal policy is to test each component for an equal length of time and not test the system, while if the total cost of testing the components exceed that of testing the system, the system is tested and the components are not. Second, consider the case when $\delta$ is "large". In this case the interfaces are much more unreliable and since there is no way of testing the interfaces by themselves the optimal policy forces us to test the entire system.

\subsection{Using prior information on interface reliability}

We now consider the situation where prior information on $\lambda_{I}$ is available. While this information could take several forms, we consider the case where the value of $\delta$ has an upper bound. For a practitioner, it is more appealing to conduct component tests that are less expensive and complex than system level tests, even when the interfaces are imperfect, as long as it is known that the interfaces are not extremely unreliable. However, it is not obvious how this knowledge affects the optimal policy and whether there are situations in which it might be optimal to test both components as well as the system, and if so in what proportion relative to each other. It is also worth noting that when a series system with perfect interfaces was considered, the situation with prior information on individual component failure rates led to results that were quite different from the situation with no a priori knowledge [10].

In this section, we modify our definition of $\delta$ slightly. Rather than defining it to be equal to the ratio $\lambda_{I} / \lambda_{C}$ we define it as a reasonable upper bound on this ratio, i.e., we make the assumption that the a priori knowledge is of the form $\lambda_{I} \leq \delta \lambda_{C}$ where $\delta$ is some known positive number. Thus the interface failure rates could range from being zero to no more than $100 \times \delta \%$ of the combined failure rates of the individual components. There is no obvious way to specify a value for $\delta$; however, a reasonable estimate may be available from historical performance in similar systems, manufacturer's specifications or even on a subjective basis. While the results that follow are best applied to situations where good estimates are available for $\delta$, the problem of designing an optimal test plan is shown here to have a closed-form solution. This allows for an optimal plan to be developed for any value for $\delta$, and a user could thus examine plans corresponding to several reasonable values and pick one that is appealing. Furthermore, closed-form expressions also exist for the maximum Type 1 and Type 2 error probabilities for any test plan with its test times and its value of $m$. These probabilities may be computed for various values of $\delta$ as well in order to assess the effects of errors in the estimate of $\delta$. This will be illustrated with numerical examples.

Now consider the optimization problem in this case. The system reliability and the distribution of the test statistic are identical to those in Section 2.1 as is the development of the optimization problem. The only difference lies in the constraint set associated with the two "inner" subproblems (10) and (11) which would have $\lambda_{I} \leq \delta \lambda_{C}$ as opposed to $\lambda_{I}=\delta \lambda_{C}$. The problem analogous to (13), (14) and (15) is:

$$
\text { Minimize } C=c_{S} t_{S}+\left(\sum_{j=1}^{n} c_{j}\right) t_{C},
$$

subject to

$$
\begin{aligned}
& \left\{\text { Maximum } t_{S} \lambda_{I}+\left(t_{S}+t_{C}\right) \lambda_{C},\right. \text { subject to } \\
& \left.\quad\left\{\lambda_{I}+\lambda_{C} \leq-\ln R_{1} ; \lambda_{I} \leq \delta \lambda_{C} ; \lambda_{I}, \lambda_{C} \geq 0\right\}\right\} \\
& \quad \leq \phi_{m}(1-\alpha), \\
& \left\{\text { Minimum } t_{S} \lambda_{I}+\left(t_{S}+t_{C}\right) \lambda_{C},\right. \text { subject to } \\
& \left.\quad\left\{\lambda_{I}+\lambda_{C} \geq-\ln R_{0} ; \lambda_{I} \leq \delta \lambda_{C} ; \lambda_{I}, \lambda_{C} \geq 0\right\}\right\} \\
& \quad \geq \phi_{m}(\beta) .
\end{aligned}
$$

However, the solution of the above problem is more complex. In the subproblem defining the LHS of (20), it is clear that the first constraint must be binding at the optimum; if we had a vector which satisfied both constraints and the first one was inactive we could increase $\lambda_{C}$ by the amount of the excess and improve the objective (while leaving the second constraint unaffected). Thus the optimum solution has $\lambda_{C}=-\ln R_{1}, \lambda_{I}=0$, with a corresponding value of the objective given by $\left(t_{C}+t_{S}\right)\left(-\ln R_{1}\right)$; the second constraint $\left(\lambda_{I} \leq \delta \lambda_{C}\right)$ is redundant at the optimum.

Next, consider the LHS of (21). The first constraint for this subproblem must be binding at the optimum; otherwise we could reduce $\lambda_{I}$ and improve the objective (the second constraint continues to be satisfied). By substituting $\lambda_{I}=\left(-\ln R_{0}\right)-\lambda_{C}$ into the objective and the second constraint, the subproblem reduces to one of minimizing $\left(-\ln R_{0}\right) t_{S}+\lambda_{C} t_{C}$ subject to $\lambda_{C} \geq\left(-\ln R_{0}\right) /$ $(1+\delta)$ and $\lambda_{C} \geq 0$. The optimum solution to the subproblem has $\lambda_{C}=\left(-\ln R_{0}\right) /(1+\delta)$ and $\lambda_{I}=\left(-\ln R_{0}\right)$ $\delta /(1+\delta)$. The objective value is given by $\left(-\ln R_{0}\right) t_{S}+\left[\left(-\ln R_{0}\right) /(1+\delta)\right] t_{C}$.

Given the above, we may now rewrite the overall optimization problem as

$$
\text { Minimize } C=c_{S} t_{S}+\left(\sum_{j=1}^{n} c_{j}\right) t_{C},
$$

subject to

$$
t_{C}+t_{S} \leq A(m),
$$




$$
\begin{gathered}
t_{C} /(1+\delta)+t_{S} \geq B(m), \\
t_{S}, t_{C} \geq 0 .
\end{gathered}
$$

Note that since $t_{C} /(1+\delta)<t_{C}$, the above problem must have $\phi_{m}(\beta) /\left(-\ln R_{0}\right)<\phi_{m}(1-\alpha) /\left(-\ln R_{1}\right)$ for feasibility. This is identical to the conditions in the previous section and thus we are guaranteed that the problem is feasible for all $m \geq m^{*}$ where $m^{*}$ is given as before by (18). Once again, it is clear that constraint (24) must hold as an equality at the optimum; otherwise we may decrease $t_{C}$ to make the constraint hold as an equality and improve the objective. Thus

$$
t_{C}=(1+\delta)\left[B(m)-t_{S}\right] .
$$

Note that since $t_{C}$ is restricted to be nonnegative, (25) also implies that $t_{S} \leq B(m)$.

Using (25) to substitute for $t_{C}$ into (22) and (23) and incorporating the upper bound for $t_{S}$ reduces the optimization problem to the following:

$$
\begin{aligned}
\text { Minimize } C= & (1+\delta)\left(\sum_{j} c_{j}\right) B(m) \\
& +t_{S}\left[c_{S}-(1+\delta)\left(\sum_{j} c_{j}\right)\right]
\end{aligned}
$$

subject to

$$
\begin{gathered}
t_{S} \geq\{(1+\delta) B(m)-A(m)\} / \delta, \\
t_{S} \leq B(m), \\
t_{S} \geq 0 .
\end{gathered}
$$

Note that although $B(m)<A(m)$ for feasible $m$, the quantity within the parentheses in the RHS of (27) could be positive or negative depending on the magnitude of $\delta$. Furthermore, the optimum value of $t_{S}$ and hence of $t_{C}$ will also depend on whether the quantity $\left[c_{S}-(1+\delta)\right.$ $\left.\left(\sum_{j} c_{j}\right)\right]$ is positive or negative, i.e., on whether $c_{S} \geq(1+\delta)\left(\sum_{j} c_{j}\right)$ or not. Note that this is the same expression that was evaluated in Section 2.1. We therefore consider the following exhaustive cases.

Case 1. $c_{S} \leq(1+\delta)\left(\sum_{j} c_{j}\right)$. Note that since the coefficient of $t_{S}$ is nonpositive in the objective, the optimum solution will have $t_{S}$ at its upper bound given by (28) while the lower bound (27) is irrelevant. Thus at the optimum, $t_{S}=B(m)$ and from $(25), t_{C}=0$, i.e., only the system is tested. The value of the objective at the optimum is $c_{S} B(m)$. Furthermore, since $B(m)$ continuously increases with $m$, the optimum value of $m$ is given by its smallest feasible value, i.e., $m^{*}$ via (18).

Case 2. $c_{S} \geq(1+\delta)\left(\sum_{j} c_{j}\right)$. Here we consider two further possibilities based on the value of $m^{*}$.

Case 2a. $\left\{(1+\delta) B\left(m^{*}\right)-A\left(m^{*}\right)\right\} \leq 0$. First note that constraint (27) is redundant here since its RHS is non- positive. Since $t_{S}$ has a nonnegative coefficient in the objective that is being minimized the optimum solution has $t_{S}=0$ and from (25) it follows that $t_{C}=(1+\delta) B\left(m^{*}\right)$, i.e., only the components are tested. The value of the objective at the optimum is $(1+\delta)\left(\sum_{j} c_{j}\right) B\left(m^{*}\right)$. To show that $m^{*}$ is optimal, first note that for this case $(1+\delta) \leq A\left(m^{*}\right) / B\left(m^{*}\right)$. Further, it may be shown that $A(m) / B(m)$ is strictly increasing in $m$ (with $\left.\lim _{m \rightarrow \infty} A(m) / B(m)=-\ln R_{0} /-\ln R_{1}\right)$. Thus it is clear that $(1+\delta) \leq A(m) / B(m)$, i.e., $\{(1+\delta) B(m)-A(m)\} \leq 0$ for all $m>m^{*}$. Hence the optimum value of $C$ is again equal to $(1+\delta)\left(\sum_{j} c_{j}\right) B(m)$ for all such $m$. However, $B(m)$ continuously increases with $m$ so that the optimum value of $m$ is the smallest feasible one, namely $m^{*}$.

Case $2 b .\left\{(1+\delta) B\left(m^{*}\right)-A\left(m^{*}\right)\right\}>0$. The RHS of (27) is now a nonnegative quantity and serves as a lower bound on $t_{S}$. Since $t_{S}$ has a nonnegative coefficient in the objective the optimum solution has $t_{S}$ at this lower bound, i.e., $t_{S}=\left\{(1+\delta) B\left(m^{*}\right)-A\left(m^{*}\right\} / \delta\right.$. Substituting this value for $t_{S}$ into (25) yields $t_{C}=\left\{A\left(m^{*}\right)-B\left(m^{*}\right)\right\}$ $((1+\delta) / \delta)$. Thus the system and the components are both tested here. Substituting the preceding value for $t_{S}$ into (26) yields an optimum objective value of

$$
\begin{aligned}
& (1+\delta)\left(\sum_{j} c_{j}\right) B\left(m^{*}\right)+\left[c_{S}-(1+\delta)\left(\sum_{j} c_{j}\right)\right] \\
& \quad \times\left[(1+\delta) B\left(m^{*}\right)-A\left(m^{*}\right)\right] / \delta .
\end{aligned}
$$

The determination of the optimal value for $m$ is more complicated here. First note that for any feasible $m$, (29) may be rearranged so that it is of the form $\left\{k_{1}+k_{2}[(1+\delta)-A(m) / B(m)]\right\} B(m)$ where $k_{1}$ and $k_{2}$ are positive constants. Now $A(m) / B(m)$ continuously increases with a limiting value of $-\ln R_{0} /-\ln R_{1}$ while $B(m)$ continuously increases in $m$ and is unbounded from above. Thus the following lemma holds:

Lemma 1. As long as $[(1+\delta)-A(m) / B(m)]$ remains positive, the value of (29) will either increase monotonically with $m$, or it will initially decrease to some minimum value and then increase monotonically with $\mathrm{m}$.

The determination of the optimum value of $m$ will depend on $\delta$. If $\left(-\ln R_{0} /-\ln R_{1}\right) \leq(1+\delta)$, then $A(m) / B(m)$ is bounded from above by $(1+\delta)$ for all feasible $m$, so that $[(1+\delta)-A(m) / B(m)]>0$ for all $m>m^{*}$. Thus we evaluate (29) at successively larger values of $m$ starting with $m^{*}$ and stop as soon as the value of (29) reaches a minimum and starts to increase. In most cases when $\delta$ is relatively small, the very first value $\left(m^{*}\right)$ will be the optimum one.

If $\left(-\ln R_{0} /-\ln R_{1}\right)>(1+\delta)$, then as the value of $m$ increases from $m^{*}$ the ratio $A(m) / B(m)$ will exceed $(1+\delta)$ for some value of $m$, say $m^{1}$. The quantity $[(1+\delta)$ 
$B(m)-A(m)]$ becomes negative for $m=m^{1}$ and remains negative for all $m>m^{1}$. To clarify this we define

$$
m^{1}=\operatorname{Inf}\left\{m \mid m>m^{*},[(1+\delta) B(m)-A(m)]<0\right\} .
$$

Thus the situation reverts to that of Case 2a for all $m \geq m^{1}$. Now consider all feasible $m$, i.e., $m \geq m^{*}$

- First consider $m$ such that $m^{*} \leq m<m^{1}$. For such $m$ the value of $[(1+\delta) B(m)-\bar{A}(m)]$ is positive and Lemma 1 applies. Thus we evaluate (29) at successively larger values of $m$ starting with $m^{*}$ and stop as soon as the value of (29) reaches a minimum and starts to increase or $m=m^{1}-1$, whichever is earlier. Let us denote the corresponding value of $m$ by $m^{0}$.

- Next consider $m \geq m^{1}$. This being Case 2a, the optimum is at $t_{C}=(1+\delta) B(m), t_{S}=0$ with an optimum value $(1+\delta)\left(\sum_{j} c_{j}\right) B(m)$ which again is strictly increasing in $m$. Therefore $m^{1}$ is the best among all such $m$.

However, there is no guarantee that the optimum value of $C$ at $m^{1}$ is better or worse than the optimum value of $C$ at $m^{0}$; this depends on the values of $\delta, c_{S}$ and $c_{j}$. From the foregoing discussion, it follows that the only candidates for the optimum value of $m$ are $m^{0}$ and $m^{1}$. Thus the corresponding objective values are evaluated at these two values and the one yielding the lower objective value is the optimum $m$. Two possibilities are shown in Fig. 1(a and b); in Fig. 1(a) the value of $m^{0}$ lies between $m^{*}$ and $m^{1}$ and the optimum value of $m$ is $m^{0}$, while in Fig. 1(b) $m^{0}=m^{*}$ and the optimum is at $m^{1}$.

In summary, we have the following results.

- Case 1: If $c_{S} \leq(1+\delta)\left(\sum_{j} c_{j}\right)$ then we use $m=m^{*}$ from (18) and test only the system for $t_{S}=B\left(m^{*}\right)$ units of time, at a total cost of $C=c_{S} B\left(m^{*}\right)$.

- Case 2a: If $c_{S} \geq(1+\delta)\left(\sum_{j} c_{j}\right)$ and $\left\{(1+\delta) B\left(m^{*}\right)-\right.$ $\left.A\left(m^{*}\right)\right\} \leq 0$, then we use $m=m^{*}$ and do not test the system at all, but test each component equally for $t_{C}=(1+\delta) B\left(m^{*}\right)$ units of time at a total cost of $C=(1+\delta)\left(\sum_{j} c_{j}\right) B\left(m^{*}\right)$

- Case 2b: If $c_{S} \geq(1+\delta)\left(\sum_{j} c_{j}\right)$ and $\left\{(1+\delta) B\left(m^{*}\right)-\right.$ $\left.A\left(m^{*}\right)\right\} \geq 0$ :

- If $\left(-\ln R_{0} /-\ln R_{1}\right) \leq(1+\delta)$ the objective is given by (29) which is evaluated successively at $m^{*}$

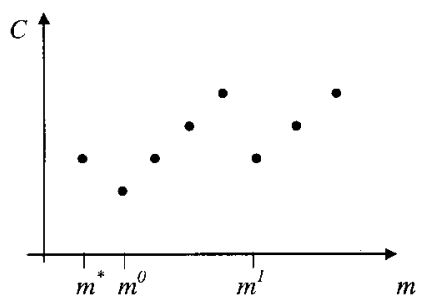

(a) optimum $m=m^{0}$

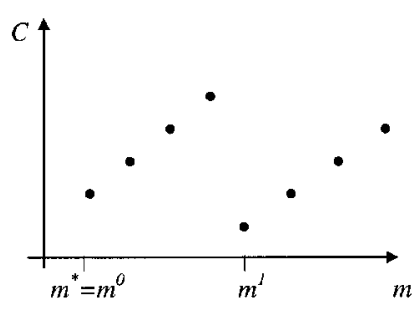

(b) optimum $m=m^{I}$
Fig. 1. Minimum test costs corresponding to different values of $m$. and larger values to find the optimum $m$, with the system and the components both being tested for $t_{S}=\left\{(1+\delta) B\left(m^{*}\right)-A\left(m^{*}\right)\right\} / \delta$ and $t_{C}=\left\{A\left(m^{*}\right)-\right.$ $\left.B\left(m^{*}\right)\right\}((1+\delta) / \delta)$ units of time respectively.

- If $\left(-\ln R_{0} /-\ln R_{1}\right)>(1+\delta)$ the candidate solutions are: (i) the value $m^{0}$ in the range $m^{*}$ to $m^{1} 1$ that minimizes (29) with system as well as component testing, and (ii) the value $m^{1}$ evaluated via (30) for which the value of the objective is $(1+\delta)\left(\sum_{j} c_{j}\right) B\left(m^{1}\right)$ and we do only component testing. The optimum value of $m$ is the one that yields the lower value for $C$, and thus if use $m^{0}$ we test both the system as well as the components according to the times in the previous paragraph, and if we use $m^{1}$ we test only the components for $t_{C}=(1+\delta) B\left(m^{*}\right)$ units of time.

\subsection{Estimating maximum type 1 and type 2 error probabilities}

An issue that is of interest is the maximum Type 1 and Type 2 error probabilities that are associated with a test plan that is derived using the procedure above. This issue becomes particularly important for assessing the degree of protection provided by sampling plans that are obtained using an incorrect value for $\delta$. These are readily computed. First note the values of $\lambda_{I}$ and $\lambda_{C}$ that solve the subproblems given by (20) and (21) are the ones that yield the maximum error probabilities. For Type 1 error (20) these were given by $\left\{\lambda_{C}=-\ln R_{1}, \lambda_{I}=0\right\}$ and for Type 2 error (21) by $\left\{\lambda_{C}=-\ln R_{0} /(1+\delta), \quad \lambda_{I}=\right.$ $\left.\left(-\ln R_{0}\right) \delta /(1+\delta)\right\}$. Substituting these into (7) to obtain $\Lambda$, and substituting this value of $\Lambda$ into (5) and (6) yields

$$
\begin{aligned}
& \text { Maximum Type } 1 \text { Error Probability } \\
& \quad=1-F_{m}\left[\left(t_{S}+t_{C}\right)\left(-\ln R_{1}\right)\right] . \\
& \text { Maximum Type } 2 \text { Error Probability } \\
& \quad=F_{m}\left[\left\{t_{S}+t_{C} /(1+\delta)\right\}\left(-\ln R_{0}\right)\right] .
\end{aligned}
$$

These error probabilities hold for any plan with its specified values of $t_{S}, t_{C}$ and $m$ and thus (31) and (32) may be used to evaluate the performance of any such plan.

The following numerical examples illustrate the above results.

\section{Numerical examples}

Example 1. Here we fix the value of $\delta$ and see how the optimum policy varies as the system test cost changes relative to the component test costs. Consider a series system of five components to be tested with $R_{0}$ and $R_{1}$ equal to 0.80 and 0.95 respectively, while the maximum acceptable probabilities of Type 1 and Type 2 error are 
specified as $\alpha=\beta=0.05$. Suppose $\delta=0.1$ and costs per unit time on test $\left(c_{i}\right)$ for components 1 through 5 are 10 , $15,5,5$ and 2 respectively; thus $\left(\sum_{j} c_{j}\right)$ is equal to 37 . For this data set the smallest value of $m$ for which $\phi_{m}(1-\alpha) / \phi_{m}(\beta)$ exceeds $\left(-\ln R_{1}\right) /\left(-\ln R_{0}\right)=0.23$ is equal to 5 (with $\phi_{5}(0.95) / \phi_{5}(0.5)=2.613 / 10.513=$ $0.25)$. Thus $m^{*}=5, A\left(m^{*}\right)=50.94$ and $B\left(m^{*}\right)=47.11$.

Case 1. Suppose $c_{S}<40.7$ so that $c_{S}<(1+\delta)\left(\sum_{j} c_{j}\right)$. Then the optimum value of $m$ is given by $m^{*}=5, t_{C}=0$ and $t_{S}=B(5)=47.11$ with a total test cost of $C=$ $47.11 c_{S}$ units.

Case 2. Suppose $40.7<c_{S}$ so that $c_{S}>(1+\delta)\left(\sum_{j} c_{j}\right)$. Here $\quad\left\{(1+\delta) B\left(m^{*}\right)-A\left(m^{*}\right)\right\}=\{(1.1)(47.11)-50.94\}$ $>0$ the situation corresponds to Case $2 \mathrm{~b}$. Furthermore, $\left(-\ln R_{1} /-\ln R_{0}\right)=4.35>(1+\delta)=1.1$, so that we need to find $m^{1}$ and $m^{0}$. Since $A(6)=\phi_{6}(0.95) /$ $-\ln 0.95=63.94$ and $B(6)=\phi_{6}(0.05) /-\ln 0.80=$ 53.062, $\{(1+\delta) B(6)-A(6)\}=-5.5<0$. Thus it follows from (29) that $m^{1}=6$. Thus the only possible value for $m^{0}$ is 5 and we evaluate the objective at the optimum times corresponding to $m^{0}=5$ and $m^{1}=6$. For $m^{0}=5$, we have $t_{S}=\{(1+\delta) B(5)-A(5)\} / \delta=8.81$ and $t_{C}=$ $\{A(5)-B(5)\}((1+\delta) / \delta)=42.13$. Thus the total cost is $C=c_{S} \times 8.81+37 \times 42.13=8.81 c_{S}+1558.81 \quad$ units. With $m^{1}=6$, we have $t_{C}=(1+\delta) B(6)=58.38$ and $t_{S}=0$. Thus the total cost is $C=37 \times 58.38=2160.06$ units. Comparing these two values for $C$ it may be seen that there are two possibilities:

- If $40.7<c_{S}<68.2$ then the optimum solution has $m=5, t_{S}=8.81$ and $t_{C}=42.13$, with a total cost of $C=8.81 c_{S}+1558.81$ units.

- If $c_{S}>68.2$ then the optimum solution has $m=6$ with $t_{S}=0$ and $t_{j}=58.38$ for all $j$, with a cost of 2160.06 units.

Similar results hold for other values of $\delta$ as well. It should be noted that for $\delta<0.0813$ the value of $\{(1+\delta) B(5)-A(5)\}$ is negative and corresponds to Case $2 \mathrm{a}$, so that in this range, one would move from pure system tests to pure component tests directly regardless of the value of $c_{S}$. In summary, when $c_{S}$ is relatively small (Case 1), we test only the system with the total cost increasing linearly with $c_{S}$. When $c_{S}$ becomes larger (Case 2 ), for intermediate values both the system and the components are tested with the total cost increasing as a piece-wise linear function of $c_{S}$, and for large values of $c_{S}$ we test only the components and the total cost is independent of system test cost. Figure 2 illustrates this for $\delta=0.1$ considered above, as well as for values of 0.01 , 0.25 and 0.5 .

Example 2. In the second illustration, we examine the effect of different values of $\delta$ on the minimum cost for fixed values of $c_{S}$ and $c_{j}$. We use the component cost

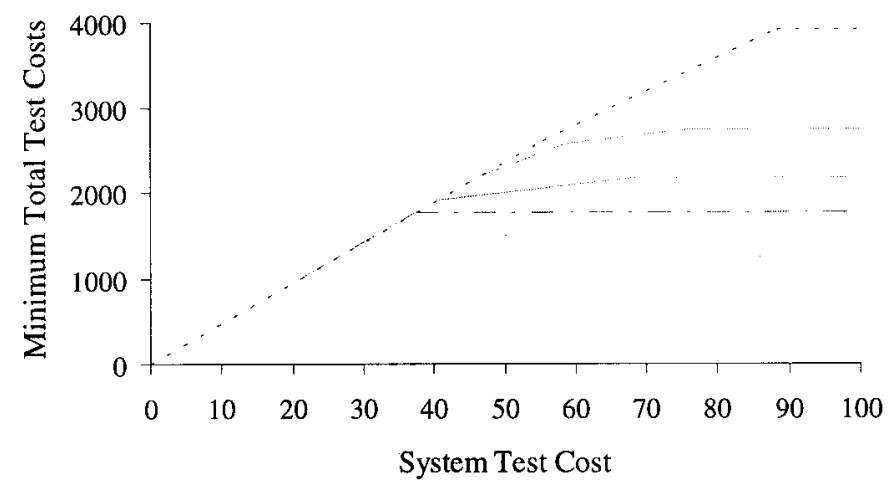

Fig. 2. Minimum total test cost as a function of system test costs.

data as in the previous example, but fix $c_{S}=65$; the values of $R_{0}, R_{1}, \alpha$ and $\beta$ are the same as before. There are three ranges of values for $\delta$ to consider here.

- First, $c_{S}<(1+\delta)\left(\sum_{j} c_{j}\right)$ as long as $\delta>0.7568$. This range for $\delta$ corresponds to Case 1 with $m^{*}=5, t_{C}=0$ and $t_{S}=B(5)=47.11$. The total test cost is $C=3062.15$ units.

- Second, $\quad c_{S}>(1+\delta)\left(\sum_{j} c_{j}\right)$ and $(1+\delta) B\left(m^{*}\right)-$ $A\left(m^{*}\right) \leq 0$ as long as $\delta \leq 0.0813$. This range for $\delta$ corresponds to Case $2 \mathrm{a}$ and the optimum solution has $m^{*}=5$, $t_{S}=0$ and $t_{C}=(1+\delta) B\left(m^{*}\right)=(1+\delta) 47.11$ units, with a total cost of $C=1743.07(1+\delta)$ units.

- Third, suppose $0.0813 \leq \delta \leq 0.7568$. These values of $\delta$ correspond to Case $2 \mathrm{~b}$. However, depending upon the specific value of $\delta$ the value of $m^{1}$ as given by (30) will vary and the solution could either involve testing of both components as well as the system (if $m=m^{0}$ ) or it may be more economical to test only the components (if $m=m^{1}$ ); the better choice may be found by simply substituting the value for $\delta$ into the appropriate cost expressions and comparing the two.

Figure 3 shows the minimum total test cost as a function of $\delta$, and as may be seen there is an initial linear region (for $\delta \leq 0.0813$ ) and a final flat region (for $\delta \geq 0.7568)$; in between, the minimum cost continuously increases with $\delta$ but the shape of the cost curve is irregular.

Example 3. Finally, we illustrate the effect of uncertainty in the estimate for $\delta$ on the maximum Type 1 and Type 2 error probabilities associated with a plan, as given by (31) and (32); clearly, these probabilities depend on $t_{C}, t_{S}$ and $m$. Consider a system with data identical to that in Example 2, and suppose $\delta=0.30$. It may be verified that the situation corresponds to Case $2 \mathrm{~b}$, and the optimal plan has $m=6, t_{C}=47.58$ and $t_{S}=16.47$ with a cost of 2831.01. Using these along with (31) and (32) yields a maximum value of 0.05 for both Type 1 and Type 2 errors. 


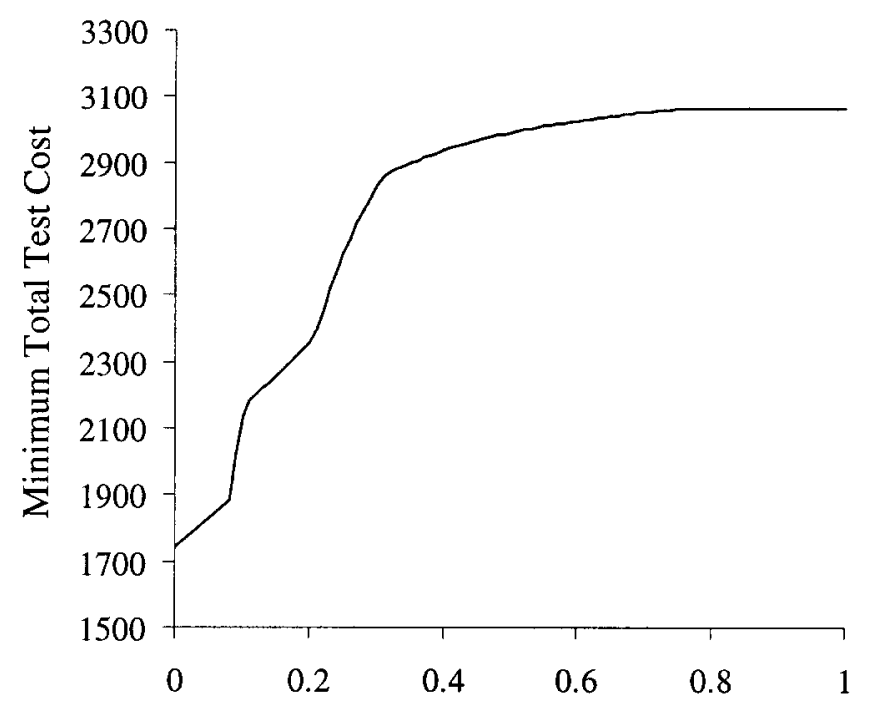

Fig. 3. Minimum total test cost as a function of $\delta$.

Now suppose that the value of $\delta$ was in error and its true value was different from 0.3 we consider errors of 10 , 20,50 and $100 \%$ in both directions and these alternate values are listed in the first column of Table 1. Columns 2 and 3 list the optimum component and system test times corresponding to each value of $\delta$, and Column 4 the corresponding value of $m$. Substituting these into (31) and (32) yields the maximum Type 1 and Type 2 error probabilities listed in Columns 5 and 6. First, note that as long as $m$ does not change, Type 1 error remains unaffected since (31) does not involve $\delta$. However, when $m$ changes, the probability of Type 1 error increases. On the other hand, Type 2 error is always affected since (32) involves $\delta$. However, for this example it may be seen that even when there is a $100 \%$ error in the estimate of $\delta$, the maximum Type 2 error probability is still only slightly more than the specified value of 0.05 .

In general, (31) and (32) may be used to compute these probabilities for various reasonable value of $\delta$ when its true value is unknown.

Table 1. Maximum Type 1 and 2 error probabilities for various $\delta$

\begin{tabular}{lccccc}
\hline$\delta$ & $t_{c}$ & $t_{S}$ & $m$ & $\begin{array}{c}\text { Max Pr } \\
\text { Type 1 } \\
\text { error) }\end{array}$ & $\begin{array}{c}\text { Max Pr } \\
\text { (Type 2 } \\
\text { error) }\end{array}$ \\
\hline 0 & 47.11 & 0 & 5 & 0.115 & 0.005 \\
0.15 & 61.03 & 0 & 6 & 0.05 & 0.027 \\
0.24 & 56.73 & 7.32 & 6 & 0.05 & 0.040 \\
0.27 & 51.65 & 12.40 & 6 & 0.05 & 0.045 \\
$\mathbf{0 . 3 0}$ & $\mathbf{4 7 . 5 8}$ & $\mathbf{1 6 . 4 7}$ & $\mathbf{6}$ & $\mathbf{0 . 0 5}$ & $\mathbf{0 . 0 5}$ \\
0.33 & 15.44 & 35.50 & 5 & 0.115 & 0.025 \\
0.36 & 14.47 & 36.47 & 5 & 0.115 & 0.028 \\
0.45 & 12.34 & 38.60 & 5 & 0.115 & 0.038 \\
0.60 & 10.21 & 40.73 & 5 & 0.115 & 0.056 \\
\hline
\end{tabular}

\section{Summary}

This paper represents the first effort to combine system level tests with component tests in the demonstration of system reliability. Such an approach is highly desirable from the perspective of a practitioner because of the often inherent unreliability of the interfaces within the system, and it represents a significant addition to the existing research in the area of component testing which has thus far addressed neither the issue of interfaces nor that of combined system and component tests. The results obtained are intuitively attractive. For a given value of $\delta$, if the cost of testing the system is relatively small, then this tends to result in testing only the system, while if it is relatively large we test only the components. For intermediate values, both the system as well as the component may be tested. Conversely, for a given cost structure, if $\delta$ is relatively small (implying that the system interfaces are reliable) we test only the components while larger values result in only system testing. With intermediate values both may be tested, unless the system test costs are very high, in which case only the components are tested. It is also worth noting that in instances where it is optimal to do only component testing, the test times with imperfect interfaces exceed the test times for a system with perfect interfaces by a factor $(1+\delta)$. This is a consequence of the fact that the latter situation is a special case of the former corresponding to $\delta=0$. Finally, if the a priori information on the interface failure rate is available in the form of an absolute upper bound, i.e., of the form $\lambda_{I} \leq u$ it is easy to show that if $u \geq-\ln R_{0}$ the bound is too large to be of use and the optimum policy calls for pure system tests with $t_{S}=B\left(m^{*}\right)$ units of time, while if $u<-\ln R_{0}$, this is exactly equivalent to solving the problem with a value of $\delta$ that is equal to $u /\left\{u+\left(-\ln R_{0}\right)\right\}$.

The results of the test plan can also offer some insight into the choice of a value for $\delta$. To illustrate this point, suppose that in Example 1 of Section 3 (where we assumed $\delta=0.1$ ) there were a total of four failures observed, two at the system level (in the 8.81 time units of system testing) and two at the component level (in the 42.13 time units of component testing). Since we have fewer than $m$ failures the sum rule used herein would accept the system. However, one would be reluctant to accept the system because of the fact that there are as many system failures as there are component failures. In fact, if we estimate $\lambda_{C}$ by $0.0475(=2 / 42.13)$ and $\lambda_{I}+\lambda_{C}$ by $0.227(=2 / 8.81)$, then $\lambda_{I} / \lambda_{C}=3.78$ which is much bigger than 0.1. Clearly, this implies that the estimate of 0.1 for $\delta$ was far too optimistic and one would have to revise this upward suitably and solve the problem with the new value of $\delta$.

There are several avenues for future research. The most obvious follow-up to this work would be the development of plans for other configurations (e.g., parallel, seriesparallel, parallel-series, $k$-out-of- $n$ etc.), and other failure 
time distributions (e.g., Gamma, Weibull). The specification of an appropriate plan and acceptance rule for each case will be a challenging task. Second, one may also examine other forms of a priori information such as simple upper bounds on the interface failure rates as well as knowledge about individual component failure rates and how these may be combined. Finally, the examination of issues such as censoring, as well as other test statistics and acceptance rules is also an open issue for further research.

\section{Acknowledgements}

The authors would like to acknowledge the constructive suggestions made by the associate editor and two anonymous referees.

\section{References}

[1] Anon. (1987) Reliability test methods, plans, and environments for engineering development, qualification, and production. Report MIL-HDBK-781D. Department of the Navy, Space and Naval Warfare Systems Command, Washington DC.

[2] Rajgopal, J. and Mazumdar, M. (1995) Designing component test plans for system reliability via mathematical programming. International Journal of Reliability, Quality and Safety Engineering, 2(1), 35-48.

[3] Easterling, R.G., Mazumdar, M., Spencer, F.W. and Diegert, K.V. (1990) System based component test plans and operating characteristics: binomial data. Technometrics, 33, 287-298.

[4] Gal, S. (1974) Optimal test design for reliability demonstration. Operations Research, 22, 1236-1244.

[5] Mazumdar, M. (1977) An optimum procedure for component testing in the demonstration of series system reliability. IEEE Transactions on Reliability, R-26, 342-345.

[6] Mazumdar, M. (1980) An optimum procedure for a series system with redundant subsystems. Technometrics, 22, 23-27.

[7] Yan, J.H. and Mazumdar, M. (1986) A comparison of several component testing plans for a series system. IEEE Transactions on Reliability, R-35, 437-443.

[8] Yan, J.H. and Mazumdar, M. (1987) A comparison of several component testing plans for a parallel system. IEEE Transactions on Reliability, R-36, 419-424.

[9] Rajgopal, J. and Mazumdar, M. (1988) A type-II censored, log test-time based component testing procedure for a parallel system. IEEE Transactions on Reliability, 37, 406-412.
[10] Rajgopal, J. and Mazumdar, M. (1995) Designing component test plans for series system reliability via mathematical programming. Technometrics, 37 (2), 195-212.

[11] Rajgopal, J. and Mazumdar, M. (1996) A system based component test plan for a series system, with type-II censoring. IEEE Transactions on Reliability, 45 (3), 375-378.

[12] Altinel, K.I. (1992) The design of optimum component test plans in the demonstration of a series system reliability. Computational Statistics and Data Analysis, 14 (3), 281-292.

[13] Altinel, K.I. (1994) The design of optimum component test plans in the demonstration of system reliability. European Journal of Operational Research, 78, 318-333.

[14] Denson, W. (1997) New system reliability assessment methods. Report of Project No. A06839, Department of Defense, Reliability Analysis Center, Rome, NY.

[15] Murphy, B. and Gent, T. (1995) Measuring system and software reliability using an automated data collection process. Quality and Reliability Engineering International, 11 (5), 341-353.

[16] Easterling, R. G. and Prairie, R. R. (1971) Combining component and system information, Technometrics, 13, 271-280.

[17] Rajgopal, J., Mazumdar, M. and Savits, T.H. (1994) Some properties of the Poisson distribution with an application to reliability testing. Probability in the Engineering and Informational Sciences, 8, 345-354.

\section{Biographies}

Jayant Rajgopal holds a Ph.D. in Industrial and Management Engineering from the University of Iowa and is currently Associate Professor of Industrial Engineering at the University of Pittsburgh. His research interests are in applied mathematical programming, reliability and production \& operations analysis. He is a senior member of IIE, a member of INFORMS and a registered professional engineer in the state of Pennsylvania.

Mainak Mazumdar holds a Ph.D. in Applied Statistics and Probability from Cornell University and is currently Professor of Industrial Engineering at the University of Pittsburgh. Prior to his current appointment he was employed as a research scientist in the Mathematics Department and in the Systems Sciences Department at the Westinghouse R\&D Center for 15 years. His research interests are in reliability and industrial statistics. He is a member of IIE, IEEE and ASA.

Subba Rao Majety is a doctoral student in the Industrial Engineering Department at the University of Pittsburgh. He has Masters degrees in Civil Engineering and Industrial Engineering from the University of Windsor. His current research is focused on the application of integer programming to reliability allocation. He is a student member of IIE and INFORMS.

Contributed by Reliability, Modeling and Optimization Department. 